Notre Dame Journal of Formal Logic

Volume XIV, Number 3, July 1973

NDJFAM

\title{
A STRONGER DEFINITION OF A RECURSIVELY INFINITE SET
}

\author{
CHARLES H. APPLEBAUM
}

1 Introduction. The purpose of this announcement is to strengthen the definition of a recursively infinite set as defined by Dekker and Myhill in [2]. This can be done after we have proved that any function that maps an immune set, $\alpha$, one-to-one into itself and has a partial recursive extension must be an $\omega$-permutation of $\alpha$.

2 Preliminaries. Let $\varepsilon$ stand for the set of nonnegative integers (numbers), $V$ for the class of all subcollections of $\varepsilon$ (sets), and $\mathcal{F}$ for the set of all mappings from a subset of $\varepsilon$ into $\varepsilon$ (functions). If $f$ is a function, we write $\delta f$ and $\rho f$ for its domain and range respectively. The relation of inclusion is denoted by $\subset$ and that of proper inclusion by $\subsetneq$. Certain families of functions are denoted by special symbols.

$$
\begin{gathered}
\mathcal{F}_{1-1}=\{f \in \mathcal{F} \mid f \text { is one-to-one }\}, \\
\mathcal{A}=\{f \in \mathcal{F} \mid f \text { has a partial recursive extension }\}, \\
\mathcal{A}_{1-1}=\{f \in \mathcal{A} \mid f \text { has a one-to-one partial recursive extension }\} .
\end{gathered}
$$

The sets $\alpha$ and $\beta$ are recursively equivalent [written: $\alpha \simeq \beta$ ], if $\delta f=\alpha$ and $\rho f=\beta$, for some $f \in \mathcal{A}_{1-1}$.

We recall from [1], Proposition 1 that

$$
f \in \mathcal{A}_{1-1} \Leftrightarrow f, f^{-1} \in \mathcal{A} \text {, for } f \in \mathcal{F}_{1-1} \text {. }
$$

A permutation of a set $\alpha$ is an $\omega$-permutation, if $f \epsilon \mathcal{A}_{1-1}$. The reader is assumed to be familiar with the contents of [2].

3 Main Results.

Notation. For $f \in \mathcal{F}, f^{n}$ is defined for $n \in \varepsilon$, as follows: $f^{0}=i$, where $i$ is the identity function, and $f^{n+1}=f \circ f^{n}$, where $\circ$ is function composition, and $f^{n+1}$ has the appropriate domain.

Theorem 1. Let $\alpha$ be an immune set and $f \in \mathcal{F}_{1-1} \cap \mathcal{A}$ such that $\delta f=\alpha$ and $\rho f \subset \alpha$, then $f$ is an $\omega$-permutation of $\alpha$. 
Proof: Let $y \in \rho f$. Put $\beta=\left\{f^{i}(y) \mid i \epsilon \varepsilon\right\}$. Thus $\beta \subset \alpha$ and $\beta$ is r.e. Hence $\beta$ must be finite. It follows that there exist numbers $i<j$ such that $f^{j}(y)=f^{\imath}(y)$. But $f \in \mathcal{F}_{1-1}$, hence

$$
\left(f^{-1}\right)^{i} \circ f^{j}(y)=\left(f^{-1}\right)^{i} \circ f^{i}(y) .
$$

Thus $f^{j-i}(y)=y$. It follows that $f\left(f^{j-i-1}(y)\right)=y$. So $f^{-1}(y)=f^{j-i-1}(y)$, where $j-i-1 \geq 0$. Hence by putting

$$
f^{-1}(y)=f^{k}(y) \text {, where } k=(\mu n>0)\left(f^{n}(y)=y\right)-1,
$$

it is clear that $f^{-1} \epsilon \mathcal{A}$. Thus by $(*), f \epsilon \mathcal{A}_{1-1}$. But since $\alpha$ is immune, it follows that $\rho f=\alpha$. Hence $f$ is an $\omega$-permutation of $\alpha$.

Remark. We recall from [2] that a set $\alpha$ is recursively infinite (r.i.) if there is an $f \in \mathcal{A}_{1-1}$ such that $\delta f=\alpha$ and $\rho f \subsetneq \alpha$, i.e., $\alpha \simeq \beta$, where $\beta \subsetneq \alpha$. It is also known that $\alpha$ is $r$.i. if and only if $\alpha$ has an infinite r.e. subset. By using Theorem 1, we can now strengthen the definition of r.i.

Theorem 2. A set $\alpha$ is r.i. if and only if there exists an $f \in \mathcal{F}_{1-1} \cap \mathcal{A}$ such that $\delta f=\alpha$ and $\rho f \subsetneq \alpha$.

Proof: The only if part is immediate. Thus let there exist an $f \in \mathcal{F}_{1-1} \cap \mathcal{A}$ such that $\delta f=\alpha$ and $\rho f \subsetneq \alpha$. It suffices to show that $\alpha$ has an infinite r.e. subset. But if $\alpha$ has no infinite r.e. subset, then $\alpha$ is immune and by Theorem $1, \rho f=\alpha$. Since $\rho f \subsetneq \alpha$, we are done.

Remark. Theorem 1 is useful in the study of automorphisms of algebraic structures and Theorem 2 makes it easier to prove a set is not immune.

\section{REFERENCES}

[1] Dekker, J. C. E., "Infinite series of isols," Proceedings of the Symposium on Recursive Function Theory, American Mathematical Society, Providence (1962), pp. 77-96.

[2] Dekker, J. C. E., and J. Myhill, "Recursive equivalence types," University of California Publications in Mathematics (N.S.), vol. 3 (1960), pp. 67-214.

Bowling Green State University

Bowling Green, Ohio 